DOI: 10.12731/2070-7568-2020-1-108-124

УДК 338.45

\title{
АНАЛИЗ ИННОВАЦИОННОГО РАЗВИТИЯ ПРОМЫШЛЕННОГО СЕКТОРА ВЛАДИМИРСКОЙ ОБЛАСТИ ПРИ ПОМОЩИ ТЕОРИИ ГРАФОВ
}

\section{Кудряков Р.И.}

Данная статья посвящена использованию математических инструментов при проведении анализа инновачионного развития промышленного сектора в региональном аспекте. Выявлено, что внедрение инноваций положительно воздействуют на синергетический эффект при взаимодействии на сектора экономики, тем самым, выражается данное влияние на системе показателей. Однако, подробной детализации причин этимологии данных показателей практически не сформированы или вовсе отсутствуют. Поэтому, особое значение в статье уделяется практическому применению теории графов. Особенность этого метода состоит в том, что в исследованиях подобного типа он не применялся, что обозначает высокий уровень актуальности и практической значимости, так как, благодаря этому методу, возникает возможность выяснения причины возникновения ключевых проблем в отраслях промышленности региона, а также составлению необходимых рекомендаций, для быстрой нейтрализации данных сложностей.

Цель исследования: Проведение анализа инноваџионного развития региона, с применением теории графов как метода, для обнаружения ключевых проблем, при проведении детализированного анализа инновачионного развития промышленного сектора Владимирской области, с последующей разработкой мероприятий по стабилизачии вылеуказанных негативных аспектов.

Методы и методология: комплекс методов теоретического обоснования (описание, статистический анализ, сравнение, метод теории графов). 
Результаты исследования: выделены основные проблемы при помощи комплексного анализа инноваџионного развития региона с применением метода теории графов, а также разработаны рекомендации по стабилизации текущчих проблемных моментов в промылиленном секторе области.

Научная новизна: использование метода теории графов при анализе инновационного развития промышленного сектора, а также разработка мер по стабилизации проблемных аспектов в конкретной отрасли внутри сектора.

Области применения результатов: Полученные, в рамках настоящего исследования результаты иелесообразно использовать в анализе различных секторов экономики субъектов государства, а применение теории графов раскрывает детализацию и обуславливает причину возникновения конкретной проблеме при проведении исследования.

Ключевые слова: теория графов; экономический анализ; инновации; инновационный потенциил; промыиленный сектор.

\section{ANALYSIS OF INNOVATIVE DEVELOPMENT OF THE INDUSTRIAL SECTOR OF THE VLADIMIR REGION USING THE GRAPH THEORY}

\section{Kudryakov R.I.}

This article is devoted to the use of mathematical tools in the analysis of the innovative development of the industrial sector in a regional aspect. It was revealed that the introduction of innovations positively affects the synergistic effect when interacting with sectors of the economy, thereby expressing this effect on the scorecard. However, detailed elaboration of the causes of the etymology of these indicators is practically not formed or even absent. Therefore, special importance in the article is paid to the practical application of graph theory. The peculiarity of this method is that it was not used in studies of this type, which means a high level of relevance and practical importance, since, thanks to this 
method, it is possible to find out the causes of key problems in the industries of the region, as well as make the necessary recommendations, to quickly neutralize these difficulties.

Purpose: to conduct an analysis of the innovative development of the region, using the theory of graphs as a method to detect key problems, when conducting a detailed analysis of the innovative development of the industrial sector of the Vladimir region, followed by the development of measures to stabilize the above negative aspects.

Methods and methodology: a set of methods of theoretical justification (description, statistical analysis, comparison, graph theory method).

Results: The main problems are identified using a comprehensive analysis of the innovative development of the region using the graph theory method, and a list of measures has been developed to stabilize the current problem areas in the industrial sector of the region.

Scientific novelty: the use of the graph theory method in the analysis of innovative development of the industrial sector, as well as the development of measures to stabilize problematic aspects in a specific industry within the sector.

Practical implications: It is advisable to use the results obtained in the framework of this study in the analysis of various sectors of the economy of state entities, and the use of graph theory reveals detail and determines the cause of a particular problem during the study.

Keywords: graph theory; economic analysis; innovation; innovation potential; industrial sector.

\section{Введение}

В современном мире, применение вычислительной техники и новых технологий становится неотъемлемой частью прогресса, и охватывает все сферы деятельности. В особенности частое использование новшеств относится к промышленному (производственному) сектору экономики. Связано это, с постоянным изменением конъюнктуры рынка, и ключевыми потребностями у потребителей, которые являются основными факторами, склоняющими к внедрению инноваций, и производству новых товаров с оптимальными параметрами, удов- 
летворяющими цели покупателей. Тем самым обеспечивая инвестиционную привлекательность региона, и увеличивая покупательскую способность отечественных товаров [1]. В связи с вышеизложенным, можно выделить, что промышленный сектор является ключевым по значимости сегментом для государства, как на федеральном, так и на региональном уровне, в связи с получением доходности от реализации товаров и продуктов, которые выпускаются на производственных предприятиях для удовлетворения потребностей внутреннего рынка, с последующим выходом на экспорт. Поэтому необходимость в проведении анализа состояния и инновационного развития промышленного сектора, является приоритетной задачей для государства. Однако, проведение традиционного статистического обследования не указывает на происхождение возникающей проблемы, поэтому автором было предложено использовать теорию графов, как метод по выявлению причины появления проблемного аспекта за счет детального рассмотрения взаимодействий компаний и выявлению партнерских отношений, а также с поставщиками дополнительных услуг, внутри промышленного сектора региона. Исходя из вышесказанного, формируется актуальность данного исследования, а использование теории графов в этом вопросе является элементом новизны и нетипичным решением для выявления отрицательных аспектов и рекомендаций по устранению.

\section{Цель исследования}

Применение метода теории графов при проведении анализа инновационного развития промышленного сектора Владимирской области для получения его детализированного состояния, а также выявления проблемных аспектов и их причин, с последующей разработкой рекомендаций по их разрешению.

\section{Методы и методология}

Проведение детализированного анализа будет осуществляться на основе описания и статистических данных за 2018-2019 г. по данным показателям индекса производства, а также, применение срав- 
нения отраслей, систематизированного метода теории графов для выявления взаимодействий в проблемной отрасли промышленности, внутри единой секторной среды, что способствует выявлению причины возникновения проблемы на основании полученных данных.

\section{Результаты исследования}

Владимирская область входит в состав Центрального Федерального Округа (далее - ЦФО). Она граничит с Ивановской, Московской, Нижегородской, Ярославской и Рязанскими областями. Рассматривая принадлежность к секторам экономики исходя из теории ФишераКларка, с углубленной методологией Хатта и Брутто, а именно, пятисекторной структуры экономики, можно выделить что данный субъект относится к вторичному сектору (производственно-промышленному), а следовательно, основной доход получаемый областью преимущественно промышленного характера. На основе полученных данных от федеральной службы государственной статистики и опросов у представителей индустриальных производств региона, были получены следующие результаты, представленные на (рисунке 1).

Рассмотрев данную диаграмму видно, что в состоянии спада, в 2019 году находятся следующие отрасли: добыча полезных ископаемых (78,9\%), текстильная промышленность (90,3\%), целлюлозно-бумажное производство $(93,1)$ и электроприборостроительное производство с индексом $(83,9 \%)$. Остальные отрасли отраженные на рисунке 1 находятся в стабильном положении и продолжают развиваться.

Поэтому для того, чтобы определить степень, и темп развития у региона, необходимо выявление инновационного потенциала, который является необходимым фактором для стабильного и высокого экономического роста региона. Термин инновационный потенциал является спорным по его интерпретации. Например: Г.А. Краюхин считал, что инновационный потенциал (далее - ИП) является совокупностью всех ресурсных составляющих относящихся к инфраструктуре, а А.Е. Монастырский считал что ИП - это совокупность процессов нацеленных на достижение цели. Поэтому автор исследование частично соглашается с мнением вышеуказанных уче- 
ных, однако формирует свое собственное понятие, которое звучит следующим образом: это совместное рациональное использование научно-исследовательских идей и ограниченных ресурсов поддерживаемой инфраструктурным обеспечением с целью эффективной интеграции новшеств.

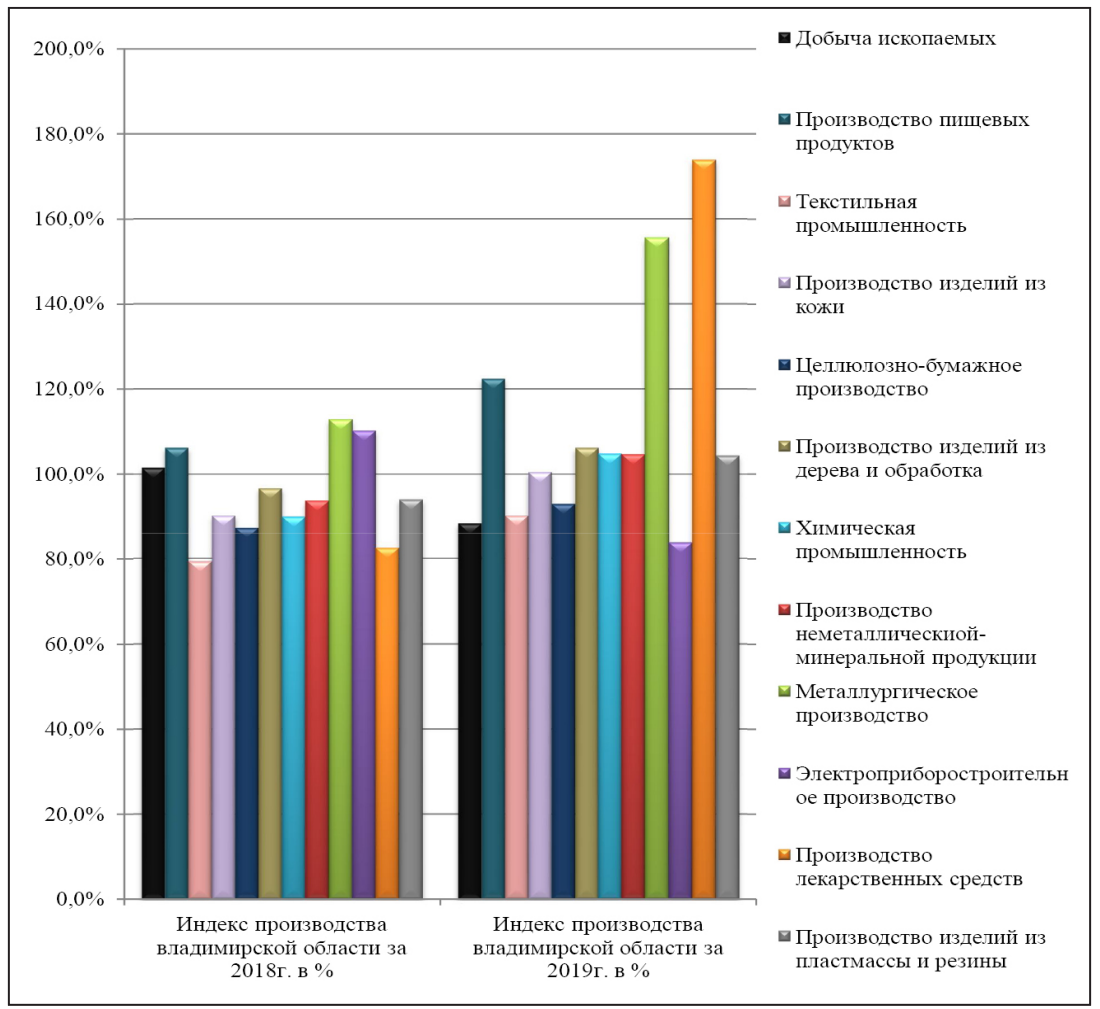

Рис. 1. Динамика индексов производства промышленного сектора Владимирской области за 2018-2019 г. [9].

Рассматривая указанный вопрос, на данный момент, для расчета инновационного потенциала является актуальной методика, представленная Независимым институтом социальной политики (далее - НИСП) [10], которая представляет собой систему числовых показателей с этапом ранжирования. Например, выделяют следующие показатели: 
- Численность работников занимающихся научно-исследовательскими и опытно конструкторскими разработками (работами) (далее - НИОКР) в регионе;

- Число выделенных ресурсов на инновации в регионе (отношение между материальными ресурсам и кадрами, направленные на реализацию инноваций;

- Количество разработанных и полученных патентов в рамках инновационной деятельности региона, и др.

На основании полученных данных инновационный потенциал рассчитывается по следующей формуле, где:

ИП - инновационный потенциал;

НП - научный потенциал;

КП - кадровый потенциал;

ТП - Технический потенциал;

ФЭП - финансово-экономический показатель;

ИКС - Информационно-коммуникационный показатель.

Таким образом, на основании данных из федеральной службы государственной статистики по Владимирской области полученных в результате запроса, был проведен следующий расчет:

Таблица 1.

Числовые значения показателей для расчета инновационного потенциала

\begin{tabular}{|l|l|}
\hline \multicolumn{1}{|c|}{ Наименование показателя } & \multicolumn{1}{|c|}{ Числовое значение } \\
\hline Научный потенциал (НП) & 12,3 \\
\hline Кадровый потенциал (КП) & 11,7 \\
\hline Технический потенциал (ТП) & 24,5 \\
\hline Финансово-экономический показатель (ФЭП) & 21,3 \\
\hline Информационно-коммуникационный показатель (ИКС) & 1,5 \\
\hline
\end{tabular}

$$
\text { иП }=\sqrt[5]{12,3 * 11,7 * 24,5 * 21,3 * 1,5}=10,24
$$

Показатель инновационного потенциала равен 10,24, что является низким индексом для региона. Рассматривая рейтинговые списки по Центральному Федеральному Округу (далее - ЦФО) по инновационному развитию Владимирская область занимает 8 место среди 18 областей входящих в состав ЦФО. Поэтому, с целью увеличения данного критерия, Администрация Владимирской области заключи- 
ла соглашения с организациями, занимающимися и оказывающими поддержку НИОКР [5, с. 40]. К таким организациям относятся: Московский государственный университет имени М.В. Ломоносова, Научно-исследовательский институт «Высшая школа экономики», Владимирский государственный университет имени А.Г. и Н.Г. Столетовых, Фонд содействия развития малых форм предприятий в научно-технической сфере, Государственная компания «Ростехнологии», Общество с ограниченной ответственностью «Владимирский инновационно-технологический центр» (филиал Фонд содействия развития малых форм предприятий в научно-технической сфере) и др.

В связи с вышесказанным, можно предположить, что использование инноваций в регионе на предприятиях промышленного сектора оказывает особое влияние на индекс производства продукции технического профиля [8, с. 56].

Стоит также отметить, что основная причина проблем связанной с низким индексом производства отражается в рамках взаимодействия объектов (компаний) в единой среде (промышленном секторе) а также поставщиками дополнительных услуг по развитию и поддержке промышленных предприятий. В рамках данного вопроса, целесообразно использовать математический метод анализа взаимодействий, теорию графов, основателем которой был математик немецкого происхождения Леонард Эйлером в 1736 г. [11, с. 16-22]. На рисунке 2. Представлены проблемные отрасли в промышленном секторе Владимирской области.

\begin{tabular}{|c|c|c|c|c|}
\hline $95.0 \%$ & \multicolumn{3}{|c|}{$93,1 \%$} & \multirow{2}{*}{$\square$ Добыча полезных ископаемых } \\
\hline 泟, & $88,5 \%$ & $90,3 \%$ & & \\
\hline $0,0 \%$ & & & $83.9 \%$ & 口Текстильная промышленность \\
\hline $0,0 \%$ & & & & \\
\hline $80,0 \%$ & & & & $\square$ Целлюлозно-бумажное \\
\hline $75,0 \%$ & & éc пр & & 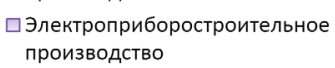 \\
\hline
\end{tabular}

Рис. 2. Сравнение индексов производства проблемных отраслей промышленного сектора Владимирской области [9]

На рисунке 2 видно, что самая проблемная отрасль промышленности в регионе, является электроприборостроительная, так как показатель индекса производства у нее самый низкий в регионе и 
показывает отрицательную динамику, что обуславливает необходимость в ее детальном рассмотрении при помощи метода теории графов и выявлению этимологии.

Поэтому, на рисунке 3. Отражено взаимодействие компаний внутри вышеуказанной отрасли.
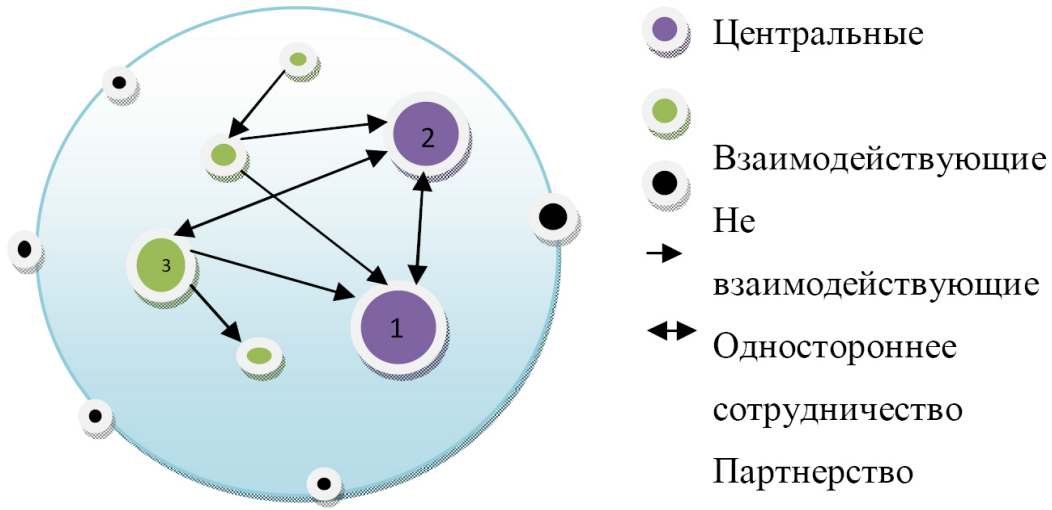

Рис. 3. Цикличный Эйлеровский направленный граф взаимодействия предприятий электроприборострительной отросли

На рисунке 3 видно[12], что сфера - это единая среда или цикл, исходя из этого и название цикличный граф. На нем также выражено 3 типа объектов, центральные: (выделенные фиолетовым цветом), у которых больше всего пересечений (ребер) с направлением, взаимодействующие: объекты которые взаимодействуют с другими компаниями в качестве поставщиков (выделены зеленым цветом), и не взаимодействующие - это компании которые не являются партнерами и поставщиками, и работают автономно. Размер объектов зависит от непосредственного объема выпускаемой продукции в регионе, чем больше выпускает продукции компания, тем больше размер данного объекта представленного на графе. Стрелочками обозначается направление от одной компании к другой, одностороннее направленное ребро означает роль поставщика к принимающему предприятию, двухстороннее направление означает партнерство в секторе и бартеру ресурсов или технологий. Под порядковыми номерами отражены предприятия области в электроприборостроитель- 
ной отрасли. Центральные предприятия, обозначенные под номером (1); и (2). К взаимодействующим объектам относятся организации с порядковыми номерами: (3); (4); (5); и (6). К невзаимодействующим объектам относятся следующие компании под порядковыми номерами: (7); (8); (9); (10); (11).

Особое внимание автор уделяет построению графа по выявлению взаимосвязи ключевых организаций электроприборостроительного производства, с поставщиками энергоэффективных технологий, инновационно-исследовательскими центрами, маркетинговыми (рекламными) компаниями, конструкторскими бюро, фондами финансовой поддержки лизинга, и отражены на рисунке 4.

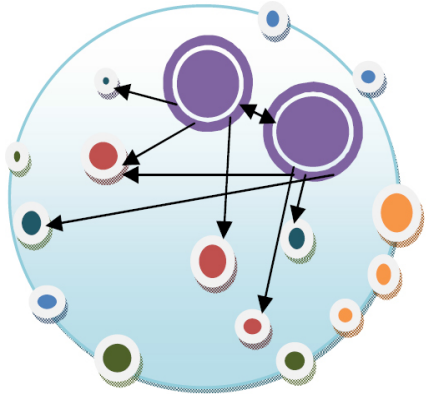

Центральные компании в отрасли

Поставщики энергоэффективных технологий

(.) Инновационно-исследовательские центры

Рекламные компании

Конструкторские бюро

Фонды финансовой поддержки и лизинга

Рис. 4. Цикличный направленный Эйлеровский граф взаимодействий центральных предприятий электроприборостроительной отрасли с поставщиками дополнительных услуг

На рисунке 4 отражены следующие виды объектов [13]:

- Центральные компании в отрасли электроприборостроения, выделены фиолетовым цветом, с порядковым номером (1) и (2);

- Поставщики энергоэффективных технологий, выделенные синим цветом, под номерами: (3), (4),(5).

- Инновационно-технологические центры выделены красным цветом, под номерами: (7), (8),(9);

- Рекламные компании выделены зеленым цветом, под номерами: (10), (11), (12);

- Конструкторские бюро выделены темно-синим цветом, представлены под номерами: (13), (14), (15); 
- Организации, представляющие финансовую поддержку и лизинг, выделены оранжевым цветом, с порядковыми номерами: (15), (16), (17).

Так же на рисунке 4 видно, что промышленные предприятия в отрасли электроприборостроения взаимодействуют с инновационно-технологическими центрами, а также с конструкторскими бюро, что положительно влияет на полученном синергетическом эффекте от внедрения инноваций в производство. Однако, они не используют в технологических процессах при изготовлении товаров энергоэффективные технологии, не используют услуги рекламных компаний и фондов по дополнительной поддержке. В связи с вышесказанным, можно выделить отрицательное влияние на производственный процесс компаний, с возникновением следующих проблем усложняющим ведение хозяйственной деятельности. К ним относятся:

- Образование высоких издержек: из-за устаревшего оборудования вследствие, высокой амортизации, и потребления энерго-коммуникативных ресурсов, а также хранения готовой нереализованной продукции;

- Недостаточность информационного продвижения товаров от производителей в данной отрасли: в связи с построением слабых маркетинговых коммуникаций усложняется выход на новые рынки и сокращается объем выпускаемой продукции, а, следовательно, сложность в реализации товаров;

- Недостаточное финансирование и низкие показатели производственной мощности: так как из-за недостатка финансовых ресурсов возникает сложность в обновлении устаревшего технического оборудования, следовательно, объем дохода от реализации не покрывает расходов и возникают высокие убытки;

- Сложность в реализации продукции: слабые и недостаточные каналы сбыта в связи с высокой конкуренцией и ослаблением количественно-качественных параметров готовой продукции.

Таким образом, индекс производства у электроприборостроительной отрасли, остается крайне низким в сравнении с другими отраслями промышленного сектора. В рамках данного вопроса ав- 
тором были сформулированы рекомендации, с целью повышения конкурентоспособности товаров предприятий Владимирской области, в электроприборостроительной отрасли и предотвращения текущих проблемных аспектов:

1. Проведение образовательных мероприятий связанных с применением элементов технологии японской системы управления проектами Р2М: данная система используется для сокращения внутренних издержек в организациях. Связано это с упразднением к эксплуатации помещениями склада хранения готовой продукции, и построением быстрого и качественного производственного процесса. В связи, с чем уменьшится уровень издержек на предприятиях, увеличиться количество и качество выпускаемого товара, что повлияет на изменение в положительную сторону индекса производства;

2. Оказать поддержку предприятиям по внедрению энергосберегающих технологий в производство: выделение субсидий для приобретения необходимых технических оснащений, которые способствуют экономии ресурсов организации;

3. Провести агитацию предпринимателей о государственных программах лизинга технического оборудования, а именно: работе специализированных лизинговых центрах, которые предоставляют высоко стоимостное техническое оснащение на правах лизинга. Способствует к увеличению количества выпускаемой продукции и уменьшит амортизационные издержки.

4. Организовать бизнес-тренинговые мероприятия направленные на построение и улучшение маркетинговых коммуникаций: данное мероприятие увеличит информированность и узнаваемость о товарах представленных данными производителями, способствует улучшению каналов сбыта и способствует росту индекса производства.

При использовании вышеописанных рекомендаций, ситуация стабилизируется, увеличится темп роста и развития данных организаций и в последствии обеспечит выход на новый рынок, и следовательно повысится индекс производства в данной отрасли. 


\section{Заключение}

При проведении исследования, было выявлено: что самый низкий индекс производства в промышленном секторе Владимирской области наблюдается у электроприборостроительной отрасли, был выявлен инновационный потенциал развития региона, который равен 10,24 что является низким значением инновационного потенциала региона. Последовательно, для выявления причины низкого индекса производства в электроприборостроительной отрасли, был применен метод теории графов, при помощи которого было установлено, что предприятия внутри отрасли не взаимодействуют с поставщиками энергоэффективных технологий, рекламными компаниями, а также с фондами финансовой поддержки и лизинга. На основании выявленных проблем, автором были предложены рекомендации по стабилизации данной ситуации в регионе, а также способствуют повышению индекса производства.

На основании вышесказанного, можно сделать вывод, о том, что метод теории графов является эффективным и тем самым, может использоваться в рамках проведения комплексного анализа инновационного развития секторов, так и экономического состояния секторов экономики, так как он является одним из методов выявления причины возникающих проблем, и показывает это детальным образом.

\section{Список литературы}

1. Аксенова Ж.Н. Оценка уровня инновационности региона и формирование условий его повышения: автореферат диссертации на соискание ученой степени кандидата экономических наук; Том. государственные унитарные системы управления и радиоэлектроники. Барнаул, 2012. 22 с.

2. Артюшина Е.В. Исследование прогнозного новшества на наличие стратегического соответствия в диверсифицированной организации // Менеджмент в России и за рубежом. 2013. № 2. С. 48.

3. Балабанов Т.И. Инновационный менеджмент. СПб.: изд. «Питер», $2011.303 \mathrm{c}$. 
4. Дежкина И.П. Инновационный потенциал хозяйственной системы и его оценка (методы формирования и оценки): учеб. пособие / И.П. Дежкина Г.А. Поташева. М.: ИНФРА-МБ. 2019. 122 с.

5. Дубинина А.В. Особенности развития инновационной экономики в регионах России // Региональная экономика: теория и практика . 2013. № 15. С. 31-38.

6. Каменских М.А. Повышение конкурентоспособности региона на основе организации инновационной инфраструктуры / М.А. Каменских, В.П. Постников // Региональная экономика: теория и практика. 2013. № 2. C. 39-43.

7. Кормановская И.Р. Структура экономики Новгородской области и ее соответствие задачам устойчивого инновационного развития // Региональная экономика: теория и практика. 2013. № 5. С. 9-21.

8. Красильщиков В.В., Осетров П.А, Кудряков Р.И. Анализ дружественных связей потребителей в социальной сети «вКонтакте» // Маркетинг в России и за рубежом. ООО «Финпресс», 2017. С. 18-29.

9. Кундиус В.А. Инновационные технологии в управлении АПК и сельскими территориями [на примере Алтайского края] // АПК: экономика, управление. 2013. Февр. (№ 2). С. 58-65.

10.Официальный сайт Федеральной службы государственной статистики по Владимирской области [Электронный ресурс]. http:// vladimirstat.old.gks.ru (дата обращения: 21.01.2020).

11. Официальный сайт Независимого института социальной политики [Электронный pecypc]. http://www.socpol.гu (дата обращения: 21.01.2020).

12. Секерин В.Д. Инновационный маркетинг: учебник. Москва: ИНФРА-М, 2018. 237 с.

13. Попадюк Т.Г. Инновационный менеджмент и экономика организаций (предприятий): Практикум / Под ред. Б.Н. Чернышева, Т.Г. Попадюк. М.: ИНФРА-М: Вузовский учебник, 2007. 240 с.

14. Фатхутдинов Р.А. Инновационный менеджмент. М.: изд. «Бизнесшкола», 2008. 448 с.

15. Эйлер Л. Введение в анализ бесконечно малых. М.: «Государственное издание», т.1, изд.2, 1961. 256 с. 
16. Goykher O.L., Skuba R.V., Bugrova O.S., Strelkov V.E., \& Kudryakov R.I. (2019). Analysis methodology of innovative development in regional industrial segment by graph theory // Lecture Notes in Networks and Systems. Vol. 57, pp. 1145-1154.

17. Goycher O., Bugrova O., \& Kudryakov R. Development of the industrial sector in the regions through innovation // E3S Web of Conferences. Vol. 110. 2019. EDP Sciences.

18. Klochkov Y., Klochkova E., Krasyuk I., Krymov S., Gasyuk D., Akobiya N. An approach to decrease the risk of losing customers // 2017 6th International Conference on Reliability, Infocom Technologies and Optimization: Trends and Future Directions, ICRITO 2017. 2018, pp. 133-142.

19. Krasyuk I.A., Bakharev V.V., Kozlova N.A., Mirzoeva D.D. Staffing in the sphere of trade: the main issues and prospects of solution// Proceedings of 2017 IEEE 6th Forum Strategic Partnership of Universities and Enterprises of Hi-Tech Branches (Science. Education. Innovations), SPUE 2017. 2018, pp. 48-50.

20. Shkurkin D.V., Krasyuk I.A., Krymov S.M., Kazantseva I.G., Zakharenko G.N. Sales policy and sales marketing system // International Journal of Applied Business and Economic Research. 2017. T. 15. № 12, pp. 203-213.

\section{References}

1. Aksenova Zh.N. Ocenka urovnya innovacionnosti regiona i formirovanie uslovij ego povy sheniya: [Abstract of dissertation for the degree of candidate of economic sciences; Tom. State unitary control systems and radio electronics]. Barnaul, 2012. 22 p.

2. Artyushina E.V. Menedzhment v Rossii i za rubezhom [Management in Russia and abroad]. OOO “Finpress". 2013. № 2, pp. 48-53.

3. Balabanov T.I. Innovacionny ’j menedzhment [Innovation Management]. SPb.: izd. «Piter», 2011. 303 p.

4. Dezhkina I.P., Potasheva G.A. Innovacionnyj potencial hozjajstvennoj sistemy i ego ocenka (metody formirovanija i ocenki) [Innovative potential of the economic system and its assessment (methods of formation and evaluation)]. M.: INFRA-MB, 2019. 122 p. 
5. Dubinina A.V. Regional'naya ekonomika: teoriya i praktika [Regional Economics: Theory and Practice]. 2013. № 15, pp. 31-38.

6. Kamenskih M.A., Postnikov V.P. Regional'naya ekonomika: teoriya i praktika [Regional Economics: Theory and Practice]. 2013. № 2, pp. 39-43.

7. Kormanovskaya, I. R. Regional'naya ekonomika: teoriya i praktika [Regional Economics: Theory and Practice]. 2013. № 5, pp. 9-21.

8. Krasil'shhikov V.V., Osetrov P.A, Kudryakov R.I. Marketing $v$ Rossii $i$ za rubezhom [Marketing in Russia and Abroad], OOO "Finpress", 2017, pp. 18-29.

9. Kundius V.A. APK: ekonomika, upravlenie [Agribusiness: economics, management]. 2013. Fevr. (№ 2), pp. 58-65.

10.http://vladimirstat.old.gks.ru

11.http://www.socpol.gu

12. Sekerin V.D. Innovatsionny marketing [Innovative marketing]. Moscow: INFRA-M, 2018. $237 \mathrm{p}$.

13. Chernyshev B.N., Popadjuk T.G. Innovatsionnyy menedzhment $i$ ekonomika organizatsiy (predpriyatiy) [Innovation Management and Economics of Organizations (Enterprises)]. M.: INFRA-M, 2007. 240 p.

14. Fatkhutdinov R.A. Innovatsionnyy menedzhment [Innovation Management]. M.: Business School, 2008. 448 p.

15. Eyler L. Vvedenie v analiz beskonechno malykh [Introduction to the analysis of the infinitesimal]. M.: State publication, V.1, 1961. 256 p.

16. Goykher O.L., Skuba R.V., Bugrova O.S., Strelkov V. E., \& Kudryakov R.I. (2019). Analysis methodology of innovative development in regional industrial segment by graph theory. Lecture Notes in Networks and Systems. Vol. 57, pp. 1145-1154.

17. Goycher O., Bugrova O., \& Kudryakov R. Development of the industrial sector in the regions through innovation. E3S Web of Conferences. Vol. 110. 2019. EDP Sciences.

18. Klochkov Y., Klochkova E., Krasyuk I., Krymov S., Gasyuk D., Akobiya N. An approach to decrease the risk of losing customers. 2017 6th International Conference on Reliability, Infocom Technologies and Optimization: Trends and Future Directions, ICRITO 2017. 2018, pp. 133-142.

19. Krasyuk I.A., Bakharev V.V., Kozlova N.A., Mirzoeva D.D. Staffing in the sphere of trade: the main issues and prospects of solution. Proceed- 
ings of 2017 IEEE 6th Forum Strategic Partnership of Universities and Enterprises of Hi-Tech Branches (Science. Education. Innovations), SPUE 2017. 2018, pp. 48-50.

20. Shkurkin D.V., Krasyuk I.A., Krymov S.M., Kazantseva I.G., Zakharenko G.N. Sales policy and sales marketing system. International Journal of Applied Business and Economic Research. 2017. V. 15. № 12, pp. 203-213.

\section{ДАННЫЕ ОБ АВТОРЕ}

Кудряков Роман Игоревич, старший преподаватель кафедры «международного права и внешнеэкономической деятельности» ФГБОУ ВО «Владимирский государственный университет имени А.Г. и Н.Г. Столетовых

ул. Горького, 87, г. Владимир, 600000, Российская Федераџия r.kudryakov-vlsu@bk.ru

\section{DATA ABOUT THE AUTHOR}

Kudryakov Roman Igorevich, Senior Lecturer, Department of International Law and Foreign Economic activity Vladimir State University named after A.G. and N.G. Stoletovs 87, Gorky Str., Vladimir, 600000, Russian Federation r.kudryakov-vlsu@bk.ru 